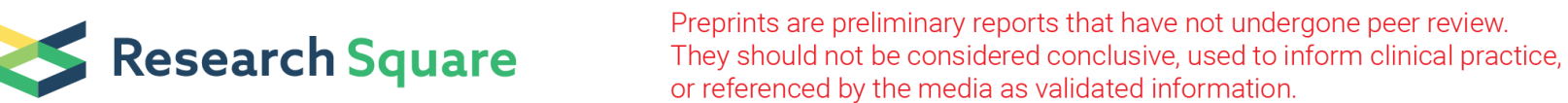

\section{A Pilot Study Using Nanoparticles and Laser Induced Photothermal Ablation to Treat Low Grade Canine Mast Cell Tumors: Evaluation of Efficacy and Safety}

\section{Lisa Parshley ( $\square$ lisa.parshley@ovccpets.com )}

Olympia Veterinary Specialist - Cancer Center and Surgical Center

\section{Lisa A. Miller}

Companion Animal Health, LiteCure LLC

\section{Luis Taboada}

Companion Animal Health, LiteCure LLC

Chelsea Tripp

Bridge Animal Referral center

\section{Scott Gustafson}

Olympia Veterinary Specialist - Cancer Center and Surgical Center

Abbey Bradley

Olympia Veterinary Specialist - Cancer Center and Surgical Center

\section{Tammy Melton}

Olympia Veterinary Specialist - Cancer Center and Surgical Center

\section{Shane Sitzman}

Olympia Veterinary Specialist - Cancer Center and Surgical Center

\section{Evan Pape}

Olympia Veterinary Specialist - Cancer Center and Surgical Center

\section{Emily Mouat}

Olympia Veterinary Specialist - Cancer Center and Surgical Center

\section{Andrew Rosenfeld}

Companion Animal Health, LiteCure LLC

\section{Research Article}

Keywords: Nanoparticles, Laser, Mast Cell Tumor, Photothermal Ablation, Cancer

Posted Date: August 26th, 2021

DOI: https://doi.org/10.21203/rs.3.rs-659087/v1 
License: (c) (i) This work is licensed under a Creative Commons Attribution 4.0 International License. Read Full License 


\section{Abstract}

Background: Nanoparticles for over forty years have been the subject of a large number of physical and bioscience research. In the last decade use of these particles in medicine has gone from theoretical to clinical trials. Passive targeting of metal-based nanoparticles takes advantage of inherent abnormalities in tumor vasculature allowing accumulation in solid tumors through a process known as the "enhanced permeability and retention" (EPR) effect. In animal tumor implant models, combination of gold and silicone nanoparticle (GSN) and exposure of tumors to laser light (at $808 \mathrm{~nm}$ ) generated enough heat to cause tumor cell death. Mast cell tumors (MCT) are the most common skin tumor in dogs, with an estimate of MCT being roughly $20 \%$ of canine skin tumors. The goal of this pilot study is to evaluate nanoparticle and laser tumor thermal ablation on low grade canine MCT model.

Results: 38 dogs with 44 mast cell tumors were enrolled in this prospective pilot study. All control tumors and those biopsied prior to laser therapy were found to be low grade MCT by histopathology (two grade method). After random number generation 8 dogs were assigned to control group and 30 dogs were assigned to treatment group. Treatment group had 36 total tumors and control group had 8 tumors present at time of enrollment. Treatment dogs had a 100\% response rate, with $94 \%$ achieving clinical remission (34 tumors). Recurrence rate was $17 \%$ in those tumors achieving clinical remission. Mean progression free time (PFT) for the treatment group was 552 days and mean PFT for the control group was 1095 days.

Conclusion: In conclusion results of this study suggest that photothermal ablation using gold-silicone nanoparticles and exposure to near infrared light $(808 \mathrm{~nm})$ provides an effective local therapy of lowgrade mast cell tumors. Median progression free time and survival was not reached in our treatment group. Suggesting that long term tumor control is possible with PTA that potentially equals surgery when margins are narrow $(<0.3 \mathrm{~cm})$ or incomplete. PTA appears to have better and more durable MCT responses than either radiation therapy and electrochemotherapy when used as sole therapies.

\section{Background}

Nanoparticles for over forty years have been the subject of a large number of physical and bioscience research. ${ }^{1}$ In the last decade use of these particles in medicine has gone from theoretical to clinical trials. ${ }^{2}$ Current biomedical research now suggests nanoparticles will in the near future provide cancer patients with targeted drug delivery, manipulation of cancer microenvironment, provide radiosensitization and chemosensitization, aid in immunotherapy, and are the basis for new methods in bioimaging. ${ }^{3-19}$.

These tiny particles get their name from their size, ranging from $1 \mathrm{~nm}$ to $>100 \mathrm{~nm}$. Construction occurs at the molecular level using chemical interactions between different compounds and elements. ${ }^{19,20,21,22}$ Based on their size, nanoparticles are, in fact, well matched to interact with biological molecules found inside and outside cells. For example, mammalian cells range in size from $2000 \mathrm{~nm}$ to 10,000 nm, cellular 
organelles are about $100 \mathrm{~nm}$ to $300 \mathrm{~nm}$, and intracellular proteins and molecules range between $10 \mathrm{~nm}$ to $50 \mathrm{~nm}^{23}$

Nanoparticles have several attributes that support their use in biomedical research and medical therapy at a microscopic level. First is their large surface area to volume ratio, allowing significantly increased chemical reactions to occur on the surface of nanoparticles. ${ }^{19} \mathrm{~A}$ second property is that they are often an appropriate size for intravascular conveyance and targeted accumulation in tumors. ${ }^{19}, 24,25$ These properties among many others give nanoparticles a potential to target and manipulate tissues at a molecular level. ${ }^{23}$

What compounds/elements used to make a nanoparticle define how they can employed in medical or cancer therapy and/or radiology. ${ }^{1-3,10,19-21}$ Nanoparticles are most often constructed using more than one compound, such as silicone and metals or lipids and glycols. Biologic behavior of a nanoparticle is dependent its molecular nature. Targeting of nanoparticles to specific tissues, such as cancer, is through active or passive mechanisms. ${ }^{1-3,11,19}$ For example, attaching ligand a to the surface of a nanoparticle will actively target it to specific cells through cell surface receptors interaction, such as transferrin or folate receptors. Nanoparticles can also be targeted by attaching monoclonal antibodies or small molecules. ${ }^{1,12,24}$

Nanoparticles made of elements or metals, such as gold, can be used to enhance radiographic imaging or be used induced to vibration with exposure to appropriate wavelengths of light. Metal-based nanoparticles in combination with laser produced light are already in pilot studies or clinical trials. Early data on this combination suggests that it is possible to perform targeted heating of tumors; achieving both coagulation of tumor vasculature and heat induced apoptosis or necrosis. ${ }^{1-4,6,9,14,17,19,26,29}$

Passive targeting of metal based nanoparticles takes advantage of inherent abnormalities in tumor vasculature (Fig. 1) allowing accumulation in solid tumors through a process known as the "enhanced permeability and retention" (EPR) effect. ${ }^{25}$ For example, a company in Houston, Texas created a gold and silicone nanoparticle (GSN) with a core of silicone and a shell of gold. Over the surface of the gold is an outer layer of polyethylene glycol (PEG).§ By including the PEG layer, GSN has an increased circulation time by reduced mononuclear phagocytic system clearance. ${ }^{28}$ After passive accumulation in tumors, exposure of these tumors to a wavelength of near infrared light $(808 \mathrm{~nm})$ causes resonance of gold atoms in the GSN shell. Reverberation of nanoparticles within the tumors causes conversion of light energy into heat energy. ${ }^{26}$

Previous research employing animal models has established that these inert GSNs are non-toxic, even at high doses. At a microscopic level there have been no toxicities noted in various organs, including the liver and spleen. The two organs responsible for clearing from circulation nanoshells not accumulated within tumors. ${ }^{27}$ 
In animal tumor implant models, combination of GSN and exposure of tumors to laser light (at $808 \mathrm{~nm}$ ) generated enough heat to cause tumor cell death. ${ }^{14}$ Near infrared radiance (NIR) has a low penetration into biological tissues. Thus, use of interstitial laser fibers are employed are required to achieve nanoparticle photothermal ablation for internal tumors. External light application will limit this therapy to superficial tumors/tissues. ${ }^{26}$

Based on initial pilot study data in various canine tumor types ${ }^{4}$, ongoing human clinical trials in prostate cancer patients ${ }^{31}$, and light-tissue interaction predictive modeling, low grade mast cell tumors were selected as our first spontaneous tumor model. Canine mast cell tumors are an excellent spontaneous tumor model in part due to their prevalence, a wealth of historical data, and a predominance of these tumors being dermal and subcutaneous. ${ }^{33}$

Mast cell tumors (MCT) are the most common skin tumor in dogs, with an estimate of MCT being roughly $20 \%$ of canine skin tumors. ${ }^{31-33,39}$ Dogs may have solitary or multiple tumors at time of diagnosis, with dogs who form one tumor being more likely to form additional tumors ${ }^{33}$ They can be located anywhere on the body but are most common in the dermis. ${ }^{31-33,39}$ Genetically any breed can be affected but there are certain breeds predisposed to forming these tumors including Boston Terriers, Pitbull, Pugs, Labradors, and Boxers. ${ }^{31-33,39}$ A majority of MCT occur in the back half of the body with a large percentage of these affecting limbs, tails, and paws. All locations where complete surgical resection may not be possible without amputation or skin flaps. ${ }^{33}$

Prognostic factors for MCT in dogs are at times controversial. Factors that appear to impact long term control of the disease include location of the tumor, ability to perform adequate surgical removal, surgical margins, number of tumors at time of diagnosis, grade (both Kiupel and Patnaik grading schemes) ${ }^{36,37}$, subcutaneous tumors, histologic pattern, multinucleation, Ki67, Ki67 + AgNOR, and KIT cellular localization pattern, and stage. Several of these prognostic factors have opposing reported data suggesting substantially different outcomes. For example, the controversy over impact of surgical margins and what defines an adequate margin. Unfortunately, no consensus has been reached on what recurrence rate can be expected for completely or incompletely resected tumors. In the literature recurrence rates range from $0-37.5 \%$ for clean margins and $12-63 \%$ for incompletely or narrowly resected $(<3 \mathrm{~mm})$ tumors. When factoring in grade, high grade (or high grade II or Grade III Patnaik) even with reasonable margins have a $40 \%$ recurrence rate. ${ }^{31-37,39-44}$ It has been mostly accepted that grade of the tumor and surgical margins achieved during resection will impact long term tumor control. $30-37,39$, $40-42$

Regardless of the prognostic value of surgical margins, a majority of MCT can be cured or have long term control with suitable local therapy $30,32,33$ Local therapies, other than surgery, are limited to radiation therapy (RTH) and electrochemotherapy. ${ }^{32,33,49} \mathrm{RTH}$ has been suggested to reduce recurrence rates after incomplete surgical margins ${ }^{45}$ and may be able to act as sole local therapy. ${ }^{46,47}$ Electrochemotherapy as an adjunctive treatment for incompletely resected MCT and as a sole local therapy has intriguing support 
in the literature. ${ }^{49,50}$ Despite these two local therapies proven efficacy and durability of response, surgical resection is still considered standard of care for canine MCT.

A pilot study testing nanoparticle and laser tumor thermal ablation on low grade canine MCT may provide an excellent opportunity to test PTA on a spontaneous tumor model and if PTA is successful the development of additional new local therapy against MCT. PTA may especially be found useful in MCT locations that are not amenable to achieving complete surgical removal. Our hypothesis in this pilot study is that photothermal ablation (PTA) using gold nanoparticles and near infrared light exposure will provide definitive therapy of low-grade mast cell tumors.

\section{Results}

38 dogs with 44 mast cell tumors were enrolled in this prospective pilot study. All control tumors and those biopsied prior to laser therapy were found to be low grade MCT by histopathology (two grade method). All tumors with only needle biopsy diagnosis had been present for over two months.

Mixed breed and Pitbull dogs were most common breed in control and treatment groups. Female dogs outnumbered males in treatment group but were in equal numbers in control group. All dogs were neutered or spayed. (see table 1)

After random number generation 8 dogs were assigned to control group and 30 dogs were assigned to treatment group. Treatment group had 36 total tumors and control group had 8 tumors present at time of enrollment. There was no significance between groups in relation to sex or breed. (see table 1)

Most tumors were in the caudal half of the dog's body. Location of the tumors differed between groups. Control group tumors were mostly truncal whereas the treatment group had a preponderance of $(>60 \%$ or 24 tumors) locations such as limbs, paws, or genital. (See table 1)

No control group tumor had progression during the month prior to surgery or after surgical removal. (see table 3) Treatment dogs had a 100\% response rate, with $94 \%$ achieving clinical remission (34 tumors). Progression was noted in 8 treatment tumors (22\% of tumors), two of which had only achieved a partial remission. Recurrence rate was $17 \%$ in those tumors achieving clinical remission. Mean progression free time (PFT) for the treatment group was 552 days and mean PFT for the control group was 1095 days. (see table 2, table 3, and Figs. 2 \& 3)

Time to complete response took on average 20 days. Toxicities in the treatment group were limited to minor dermal thermal burns/wounds, necrosis of tumor, scabs, swelling of tumor to 48 hours, and rare short-lived pain (<24 hours). All toxicities were resolved by 14 days. Antibiotic use was limited to dogs (5) who had self-trauma induced infections. All treated sites healed without long term sequelae. Control group had no toxicities noted due to nanoparticle infusion. However, two of the control dogs required reparative surgery and bandaging to allow second intention healing. (see table 3 ) 
There was no statistical difference between control and laser group in PFT $(P=0.538$ and Chi Squared 0.378). All tumors were censored because they were still clinical remission or due to death of the dog from other diseases. Median progression free period was not reached for either group. (See table 4 and Figs. 2 \& 3 ).

\section{Discussion}

The purpose of this pilot study was to evaluate the efficacy and safety of photothermal ablation (PTA) via nanoparticles and laser light $(808 \mathrm{~nm})$ against cutaneous and subcutaneous tumors. Canine mast cell tumors (MCT) were selected as the spontaneous tumor model for the study due to their prevalence, location being cutaneous and subcutaneous, and due the wealth of historical data on this cancer. The gold standard therapy for low grade MCT (two grade system - Kiupel) ${ }^{37}$ is considered surgical removal or an equivalent local therapy due to the low risk of metastasis of these tumors. ${ }^{37}$ The main finding of this study was that PTA provided effective local control of canine low grade MCT with minimal selfresolving toxicities. The responses matched and, in some cases, exceeded local control found in narrowly

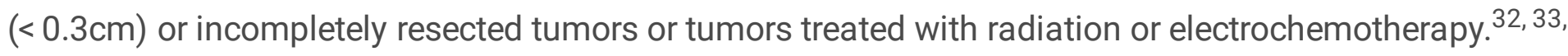
49

In our study, all PTA treated tumors responded $(\mathrm{RR}=100 \%)$ with all but two tumors achieving a clinical remission (94\% clinical remission). Two tumors not reaching clinical remission achieved PR $(>50 \%$ response). Both response and clinical remission rate exceed previously reported responses for radiation therapy (RR $=41-88 \%$ and $C R=34-59 \%$ ) and electrochemotherapy (RR-80-100\% and $C R=70 \%$ ). ${ }^{46-50}$ Caution should be taken when comparing to radiation and electrochemotherapy as the studies on these therapies included in high grade and large tumors. Whereas, in our pilot study had small tumors and no obvious high-grade tumors. Thus, apparent differences between these local therapies and PTA could be due to grade and size of the MCT in the studies. ${ }^{46-50}$

A majority of PTA treated MCT had extended progression free times (PFT), with $78 \%$ of tumors being in clinical remission at time of last follow up. Mean progression free time was 552 days. Interestingly, two of the eight tumors that had progression had only achieved a partial remission. When PFT was evaluated with just the tumors achieving clinical remission our recurrence rate it was only $17 \%$. Overall recurrence rates in our study are lower or equal to reported recurrence rates for incompletely resected MCT or tumors resected with $<0.3 \mathrm{~cm}$ margins $(12-63 \%)$. Suggesting that PTA could be equivalent to surgical resection with incomplete margins or narrow margins $(<0.3 \mathrm{~cm}) .3137,39,41-44$ When comparing PTA recurrence rates to those reported for electrochemotherapy and radiation therapy (23-50\%), PTA was more effective in establishing long term disease control. $46,47,49,50$ Once again the differences in these studies may hamper comparisons in local therapies that are not surgical.

Because of the paucity of pathologic evaluation in the animals entered into this study, we did not distinguish between cutaneous and subcutaneous tumors during statistical analysis. In previous studies subcutaneous MCT have lower chances of metastasis and similar rates of local recurrence after 
incomplete or narrowly excised tumors. ${ }^{37,52,53}$ Therefore it is the author's opinion that the data is not weakened by not distinguishing between subcutaneous or dermal tumors. Although, it should be noted that after random number assignment, all the control group tumors were dermal and freely movable. Whereas in the treatment group nine tumors were subcutaneous and five of the tumors were fixed to the deeper tissues. Such a distribution that may add bias to the results between the groups, especially as it is suggested that it is easier to achieve surgical margins with dermal tumors. ${ }^{32,42,56}$

There are several unintended biases in our study. All control group tumors surgeries were performed by a board-certified surgeon who achieved at least one facial plane for deep margins in all tumors. In MCT and other tumor types it has been previously demonstrated that having a board-certified surgeon as compared to a referring veterinarian performing tumor resections improves surgical margins and reduces local recurrence rates. ${ }^{54,55}$ Another bias potentially impacting recurrence rates for both groups was tumor size distribution between the groups. In the control group $>60 \%$ of the tumors were less than $1.0 \mathrm{~cm}$ whereas $<42 \%$ of treatment group tumors were $<1.0 \mathrm{~cm}$. Suggesting that these two groups may not be adequately sized matched for comparison.

Location of MCTs have been embroiled in long controversy involving recurrence rates and disease control with some suggesting that certain locations have shorter PFT. Most of the controversy is likely related the ability in achieving adequate surgical resection of the tumor. Our control group had tumors predominantly located on the trunk, hip, or shoulder, locations suggested to have better outcomes and lower recurrence rates. Whereas in the treatment group $>75 \%$ tumors were in the locations previously suggested to have lower disease control.

Toxicities noted in our control group were all surgical related. In the treatment study group all toxicities noted were minor and self-resolving, including minor dermal burns, necrotic tumor tissue, and some pain. All toxicities resolved by two weeks and none of the PTA sites required corrective surgery or long-term bandaging. Medical therapy for pain was rare and usually stopped within 24 hours of the laser therapy. Interestingly, two of control group animals (2/8) required bandaging/surgical wound care for $>4$ weeks due to dehiscence of surgical sites.

Another weakness of our study includes low tumor numbers for the control group. Choice of the number of controls was in large part due to the wealth of historical data on MCT after surgical resection and numerous previous nanoparticle toxicity studies. ${ }^{27}$ Our control group was able to provide confirmation of previous outcomes with surgery and toxicity studies of the nanoparticles, and that these particles did not have direct anticancer effects.

In the future it would be advantageous to ensure all tumors have a pathologic diagnosis to allow further determination of relationships between grade, cutaneous, fixed, or invasive, and subcutaneous MCTs. Our study did not require biopsies as it was desirable to include small tumors be included, tumors that might be mostly removed during a biopsy. It should be noted that all tumors which had subsequent biopsies were low grade (37) suggesting the strict inclusion requirements did select primarily low grade MCT. 
Future studies should include repeat PTA therapy with and without GSN infusions, to assess whether those tumors with only a partial response could be converted into full clinical remissions. It would be advantageous to assess if repeating PTA after recurrence could provide a second response and/or clinical remission. It will be key in such studies to assess for toxicities from second GSN infusions, ability of tumors to retain GSN particles long term, and for tumor tissue to accumulate GSN after second infusions. Answers to these questions could provide evidence the need for future infusions of GSN and what toxicities or impact of a second infusion might have on the patients and tumors.

\section{Conclusions}

In conclusion results of this study suggest that photothermal ablation using gold-silicone nanoparticles and exposure to near infrared light $(808 \mathrm{~nm})$ provides an effective local therapy of low-grade mast cell tumors. Median progression free time and survival was not reached in our treatment group. Suggesting that long term tumor control is possible with PTA that potentially equals surgery when margins are narrow $(<0.3 \mathrm{~cm})$ or incomplete. PTA appears to have better and more durable MCT responses than either radiation therapy and electrochemotherapy when used as sole therapies. Toxicities in our study were minor and resolved without surgical intervention, unlike our control group which had two dogs requiring long term supportive bandaging or surgical correction. Future studies should include more cases, of different grades of MCT, second PTA for those not fully achieving clinical remission or recurrent tumors, assessment of long term GSN retention in tumors, and evaluation of other cutaneous, subcutaneous, or easily illuminated tumor types.

\section{Methods}

From January 2016, through August 2020, we conducted a two center, prospective, parallel-group pilot study to test the safety and efficacy of nanoparticle enhanced Photothermal Ablation (nPTA) on canine mast cell tumors (MCTs). Subjects in this study were selected from patients presenting for consultation to Olympia Veterinary Specialists Cancer Center and Bridge Animal Referral Center.

Any dermal or subcutaneous low grade or suspected low-grade mast cell tumors were considered for inclusion in this prospective study. Eligibility was determined based on size, stage, appearance, duration, and presence of other diseases. Tumors had to be less than $1.8 \mathrm{~cm}$, have no bruising or ulceration, and present for more than two months. All dogs must be stage I and have no comorbidities.

All dogs were confirmed (Kiupel two grading system, ref Kiupel M) or presumed low grade based if they met all the inclusion criteria. Cytologic diagnosis of mast cell tumors prior to enrollment was considered acceptable. Cytologic or pathologic diagnosis was either achieved through board certified pathologist or a board-certified oncologist.

All dermal or subcutaneous lumps were mapped. Needle biopsies were performed on any previously undiagnosed masses. All dogs underwent staging evaluation including physical examination, abdominal 
ultrasound, and blood work. ${ }^{33}$ Signalment, tumor location, and tumor size were recorded. All tumors were as considered individual data points for analysis.

All dogs were placed on diphenhydramine ( $2 \mathrm{mg} / \mathrm{kg}$ PO q 12 hours) and famotidine $(0.5 \mathrm{mg} / \mathrm{kg} \mathrm{PO} \mathrm{q} 24$ hours). Medications were administered at home for at least a week prior to infusion of nanoparticles and laser ablation. Previous therapies or surgeries were allowed provided they were performed or administered longer than 2 weeks prior to entry into the study.

All clients were offered and declined standard of care for low grade tumors, including surgical removal. Informed consent was obtained from all clients prior to enrollment into the study. Random number generation $£$ selected dogs into control or treatment groups with a 4:1 ratio (treatment dogs to control group).

GSN nanoparticles used have a $120 \mathrm{~nm}$ silicone core and with gold shell. A polyethylene glycol (PEG) outer layer gives the nanoparticle a total size $150 \mathrm{~nm}$. PEG layer reduces the ability of mononuclear phagocytic system from clearing the GSN from circulation prior to tumor accumulation. ${ }^{4}$

All dogs had infusions of GSN $(5.2 \mathrm{ml} / \mathrm{kg})$ according to manufacturer§ instructions while monitoring temperature, heart rate, and respiratory rate. Dose of infused GSN was set at $5.2 \mathrm{ml} / \mathrm{kg} / \mathrm{dog}$ based on previous dose-escalation studies and published work on nanoparticle ablation of the canine prostate. ${ }^{4}$ Infusions were via a peripheral IV catheter, a standard IV drip set utilizing a drip chamber, and an inline TNA-1 filter $\square$ of $>5$ microns. Twenty-four (24) hours after infusion dogs were evaluated for any changes in the sentinel mass.

Control dogs received nanoparticle infusion but no laser light application. One month after infusion the tumor was measured and removed by the same American College of Surgical Veterinarian board-certified surgeon. All control tumors were submitted for histopathology.

All treatment dogs, 24 hours after infusion, had their tumor(s) measured, shaved and cleansed with chlorhexidine surgical scrub and $0.9 \%$ saline. A local anesthetic ring block was performed using bupivacaine and lidocaine (3:1 ratio) with $10 \%$ of total volume sodium bicarbonate. Lidocaine hydrochloride topical solution USP (Viscous) 2\% was applied directly to the area of the tumor to be exposed to light.

Laser safe eyewear placed over the eyes of the treated dogs. All person(s) in the treatment area wore laser safe eyewear during the procedure. The laser device ${ }^{\geq}$set to deliver an $808 \mathrm{~nm}( \pm 10 \mathrm{~nm})$ light dose. A specialized laser probe was designed to deliver a uniform irradiance beam (with a fixed laser spot area of $0.5 \mathrm{~cm}^{2}$ ) to the surface of the tumor, and efficiently wick away surface heating generated by tissue absorption. ${ }^{\alpha}$ Using the specialized laser probe, light was apply directly to the tumor surface. Light treatments ranged in radiant power from $3 \mathrm{~W}$ up to $7 \mathrm{~W}$ (resulting in an irradiance of $6-14 \mathrm{~W} / \mathrm{cm}^{2}$ ). The number of light exposures sites per tumor varied between tumors, primarily to ensure the entire tumor's surface was treated. Laser parameters were selected though the use of a predictive physics-based 
computational model[1] of light diffusion in tissue. Modeling helped predict increases in tissue temperature due to photon absorption and the presence and distribution of GSN's within the tumors after intravenous administration.

A thermal camera€ was used at 20-30 second intervals during laser treatment to ensure each treatment site reached appropriate temperature (ideally $55-65^{\bullet} \mathrm{C}$ ). If the treated site temperature did not reach $\geq 50^{\circ} \mathrm{C}$ after 1 minute of exposure, the power setting was increased incrementally by $0.5 \mathrm{~W}$ until appropriate temperature was reached. The maximum radiant power setting did not exceed $7 \mathrm{~W}$. The total treatment time and maximum temperature reached was recorded for each laser application in seconds.

Patients were recovered from anesthesia/sedation and monitored during recovery. Patients were sent home the same day as the laser procedure and were instructed to prevent licking or chewing through the use an e-collar and tee shirts where applicable. All patients were rechecked weekly for four weeks, then monthly for the duration of the study period. At each appointment, the follow information was recorded; mass size (using metric calipers), pictures of the masses, and how patients were doing at home was gathered. Mast cell tumor measurements were made by the same person at every visit.

Clinical remission, partial remission, stable disease, and progressive disease were determined in accordance with previously published VOCG consensus for these designations in solid tumors. ${ }^{38}$ Simple response rate, confidence ratio, mean progression free time, mean survival time, mean of time to response, and Kaplan-Meier survival curves evaluating progression free period, median survival were calculated using Microsoft Excel spread sheet and MedCalc®. Chi square, confidence interval, and simple t-test were calculated based on Kaplan Meier data. Significance was set at $P=0.05$.

\section{Abbreviations}

EPR: Enhanced Permeability and Retention

GSN:Gold and Silicone Nanoparticle

PEG:Polyethylene Glycol

NIR: Near infrared radiance

MCT: Mast cell tumors

RTH: Radiation Therapy

PTA:Photothermal Ablation

PFT: Progression Free Times

nPTA: Enhanced Photothermal Ablation 


\section{Declarations}

\section{Ethics Approval and consent to participate:}

The study was carried out in compliance with the ARRIVE guidelines and all methods were performed in accordance with the relevant guidelines and regulations as set forth by the International Council for Laboratory Animal Science (ICLAS). This clinical study was approved by the Olympia Veterinary Cancer Center Research Committee (Olympia, WA), the Clinical Studies Advisory Committee of Companion Animal Health (New Castle, DE), and the Veterinary Cancer Specialty Care Research Committee (Edmonds, WA). In this study, all dogs who participated were client-owned dogs and all owners received a detailed written description of the study, an explanation of the procedure(s), and written owner consent was obtained for enrollment. All dogs that participated in the study were directly overseen by a veterinarian to ensure no harm was incurred during study participation.

\section{Funding}

Authors LM, AR and LDT are currently employed by Companion Animal Health (LiteCure) and were involved in the article preparation. Funding for this study was provided by Companion Animal Health and was offered as a minimal stipend paid towards the treatment of the study participants and was offered to both control and treatment groups equally prior to randomization.

\section{Competing interests:}

All authors declare that the research was conducted in the absence of any commercial or financial relationships that could be construed as a potential conflict of interest.

\section{Consent for publication}

All authors give consent for publications

\section{Authors' contributions}

A. primary investigator and prepared manuscript and performed research. Authors C.D. secondary investigators, edited manuscript, author E. edited manuscript, secondary investigator, and performed research, author F.I performed research and review manuscript, author $\mathrm{H}$. edited and reviewed manuscript and data evaluation

\section{Availability of data and materials}




\section{Acknowledgments}

The authors gratefully acknowledge the support of the pet owners and practice staff, as well as veterinary colleagues and they acknowledge the impact of cited references on the analysis of this work. All authors provide their consent for publication. Data and materials from the study are available upon request from the corresponding author.

\section{References}

1. Bertrand N, Wu J, Xu X, et al., Cancer nanotechnology: The impact of passive and active targeting in the era of modern cancer biology. Adv Drug Deliv Rev. 2014 Feb; 66:2-25.

2. Zhang L, Gu FX, Chan JM, Wang AZ, et al., Nanoparticles in Medicine: Therapeutic Applications and Developments. Clinical pharmacology \& Therapeutics. 2008; (83) 5.

3. Jain S, Hirst D, Sullivan M. Gold Nanoparticles as Novel Agents for Cancer Therapy. The British Journal of Radiology 2012; 85: 101-113.

4. Schwartz JA, Price RE, Gill-Sharp KL, Sang KL et al., Selective nanoparticle-directed ablation of the canine prostate. Lasers Surg. Med. 2011; 43, 213-220.

5. Hirsch LR, Stafford RJ, Bankson JA, Sershen SR et al., Nanoshell-mediated near-infrared thermal therapy of tumors under magnetic resonance guidance. Proc. Natl. Acad. Sci. U.S.A. 2003; 100, 13549-13554.

6. Abadeer NS, Murphy CJ. Recent progress in cancer thermal therapy using gold nanoparticles. $J$. Phys. Chem. 2016; C 120, 4691-4716.

7. Loo C, Lin A, Hirsch L, Lee MH et al., Nanoshell-enabled photonics-based imaging and therapy of cancer. Technol. Cancer Res. Treat. 2004; 3, 33-40.

8. Rastinehead AR, Anastos H, Wajswol E, et al. Gold nanoshell-localized photothermal ablation of prostate tumors in a clinical pilot device study. PNAS 2019; 116(37): 18590-18596.

9. Stern JM, Solomonov VV, Sazykina E, Schwartz JA, Gad SC, Goodrich GP. Initial Evaluation of the Safety of Nanoshell-Directed Photothermal Therapy in the Treatment of Prostate Disease. International journal of toxicology 2015

10. Rahul J, Velpurisiva P, Gad A, et al. Targeting Strategies for the Combination Treatment of Cancer Using Drug Delivery Systems. Pharmaceutics 2017, 9(4), 46.

11. Kydd J, Jadia R, Velpurisiva P, et al. Targeting Strategies for the Combination Treatment of Cancer Using Drug Delivery Systems. Pharmaceutics. 2017; 9(4), 46.

12. Chamseddine I, Kokkolaras M. Nanoparticle optimization for enhanced targeted anti-cancer drug delivery. J Biomech Eng. 2017; Oct 16. 
13. Fred Hutchison Cancer Institute: https://www.fredhutch.org/en/news/centernews/2017/08/targeted-temporary-gene-therapy-through-nanoparticles.html

14. O'Neal DP, Hirsch LR, Halas NJ, Payne JD, West JL. Photo-thermal tumor ablation in mice using near infrared-absorbing nanoparticles. Cancer Lett. 2004; 209(2):171-6.

15. Lal S, Clare SE, Halas NJ, Nanoshell-enabled photothermal cancer therapy: Impending clinical impact. Acc. Chem. Res. 2008; 41, 1842-1851.

16. Huang X, El-Sayed IH, Qian W, El-Sayed MA. Cancer cell imaging and photothermal therapy in the near-infrared region by using gold nanorods. J. Am. Chem. Soc. 2006; 128, 2115-2120.

17. Guo L, Niu G, Zheng X, Ge J, et al. Single Near-Infrared Emissive Polymer Nanoparticles as Versatile Phototheranostics. Adv Sci (Weinh) 2017; 10:4(10).

18. Rancoule $\mathrm{C}$, Magné $\mathrm{N}$, Vallard A, et al., Nanoparticles in radiation oncology: From bench-side to bedside. Cancer Letters. 2016; (375)2: 256-262.

19. Allegra A, Penna G, Alonci A<background-color:\#DDDDDD;bivertical-align:baseline;>,</backgroundcolor:\#DDDDDD;bivertical-align:baseline;><bivertical-align:baseline;></bivertical-align:baseline;>et al. Nanoparticles in Oncology: The New Theragnostic Molecules. Anti-Cancer Agents in Medicinal Chemistry. 2011; 11: 669-686.

20. Ferrari M. Cancer nanotechnology: opportunities and challenges. Nat. Rev. Cancer. 2005; 5: 161-171.

21. Grobmyer SR, Iwakuma N, Sharma, et al., What is cancer Nanotechnology? In Cancer Nanotechnology, Methods in Molecular Biology, editors In Stephen Grobmyer and Brij Moudgil No. 624. Humana Press 2010: Springer Science + Business LLC; 2010: $p$ 1-9.

22. ASTM International. E 2456-06 Terminology for nanotechnology. West Conshohocken, PA: ASTM International, 2006.

23. Vertegel AA, Siegel RW, Dordick JS. Silica nanoparticle size influences the structure and enzymatic activity of adsorbed lysozyme. Langmuir. 2004; 20: 6800-6807.

24. Wang B, Zhai Y, Shi J, Zhuang L, et al. Simultaneously overcome tumor vascular endothelium and extracellular matrix barriers via a non-destructive size-controlled nanomedicine. J Control Release. 2017; Oct 17.

25. Maeda $\mathrm{H}$. The enhanced permeability and retention (EPR) effect in tumor vasculature: the key role of tumor-selective macromolecular drug targeting. Adv Enzyme Regul. 2001; 41:189-207.

26. Kaur P, Aliru ML, Chadha AS, Asea A, et al. Int J Hyperthermia. 2016; 32(1): 76-88.

27. Gad SC, Sharp KL, Montgomery C, Payne JD, et al. Evaluation of the Toxicity of Intravenous Delivery of Auroshell Particles (Gold-Silica Nanoshells). Intl J of Toxicology 2012; 31(6) 584-594.

28. Magaña IB, Yendluri RB, Adhikari P, et al. Suppression of the reticuloendothelial system using $\lambda$ carrageenan to prolong the circulation of gold nanoparticles. Ther Deliv. 2015; 6(7):777-83.

29. Tang F, Zhang Y, Zhang J, Guo J, Liu R. Assessment of the efficacy of laser hyperthermia and nanoparticle-enhanced therapies by heat shock protein analysis. AIP Advances 4 2014; 031334. 
30. Stern J, Solomonov V, Sazykina E. et.al. Initial Evaluation of the Safety of Nanoshell-Directed Photothermal Therapy in the Treatment of Prostate Disease. Int J Toxicol. Jan-Feb 2016;35(1):38-46

31. London C, Thamm D. Mast Cell Tumors. 5th ed. St Louis, MO: Saunders Elsevier; 2013.

32. Garrett L. Canine mast cell tumors: diagnosis, treatment, and prognosis. Vet Med (Auckl), vol. 2014; 5: $49-58$.

33. Withrow and MacEwen's Small Animal Clinical Oncology, Editors David Vail, Douglas Thamm, Julius Liptak, October 2019, Sect. 21

34. Habil M, Kiupel M, Camus, et al., Diagnosis and Prognosis of Canine Cutaneous Mast Cell Tumors. Vet Clin of NA: Small Animal Practice. 2019; 49(5): 819-836.

35. Mullins M, Dernell W, Withrow S, et al., Evaluation of prognostic factors associated with outcome in dogs with multiple cutaneous mast cell tumors treated with surgery with and without adjuvant treatment: 54 cases (1998-2004). J Am Vet Med Assoc. 2006; 228(1):91-5.

36. Patnaik AK, Ehler WJ, MacEwen EG. Canine cutaneous mast cell tumor: morphologic grading and survival time in 83 dogs. Vet Pathol. 1984;21(5):469-474.

37. Kiupel M, Webster JD, Bailey KL, et al. Proposal of a 2-tier histologic grading system for canine cutaneous mast cell tumors to more accurately predict biological behavior. Vet Pathol. 2011;48(1):147-155.

38. S M Nguyen, D H Thamm, D M Vail, C A London. Response evaluation criteria for solid tumours in dogs Veterinary Cooperative Oncology Group (VCOG) consensus document. Vet Comp Oncol 2015 Sep;13(3):176-83.

39. Śmiech A, Ślaska B, Łopuszyński W, Jasik A. et. al. Epidemiological assessment of the risk of canine mast cell tumours based on the Kiupel two-grade malignancy classification. Acta Vet Scand 2018; 60:70.

40. Cahalane AK, Payne S, Barber LG, et al. Prognostic factors for survival of dogs with inguinal and perineal mast cell tumors treated surgically with or without adjunctive treatment: 68 cases (19942002). J Am Vet Med Assoc. 2004;225(3):401-408.

41. Thompson J, Pearl DL, Yager JA, Best SJ, et. al. Canine Subcutaneous Mast Cell Tumor: Characterization and Prognostic Indices. Veterinary Pathology, 48(1) 156-168.

42. Milovancev M, Townsend KL, Tuohy JL, Gorman E, et al. Long-term outcomes of dogs undergoing surgical resection of mast cell tumors and soft tissue sarcomas: A prospective 2-year-long study. The American College of Veterinary Surgeons, Veterinary Surgery 2019; 49:96-105.

43. Weisse $C$, Shofer FS and Soremno K: Recurrence rates and sites for grade II canine cutaneous mast cell tumors following complete surgical excision. J Am Anim Hosp Assoc 38: 71-73, 2002.

44. Michels GM, Knapp DW, DeNicola DB, Glickman N and Bonney P: Prognosis following surgical excision of canine cutanous mast cell tumors with histopathologically tumor-free versus nontumorfree margins: a retrospective study of 31 cases. J Am Anim Hosp Assoc 38: 458-466, 2002. 
45. Kry KL, Boston SE. Additional local therapy with primary re-excision or radiation therapy improves survival and local control after incomplete or close surgical excision of mast cell tumors in dogs. Vet Surg. Epub January 6, 2014.

46. Dobson J, Cohen S, Gould S. Treatment of canine mast cell tumours with prednisolone and radiotherapy. Vet Comp Oncol. 2004;2(3):132-141.

47. Lejeune A, Skorupski K, Frazier S, et. al., Aggressive local therapy combined with systemic chemotherapy provides long-term control in grade II stage 2 canine mast cell tumour: 21 cases (1999-2012). Vet Comp Oncol. 2015; 13(3):267-80.

48. Carlsten KS, London CA, Haney S, et. Al., Multicenter Prospective Trial of Hypofractionated Radiation Treatment, Toceranib, and Prednisone for Measurable Canine Mast Cell Tumors. J Vet Intern Med. 2012; 26(1).

49. Cemazar M, Tamzali Y, Sersa G, Electrochemotherapy in veterinary oncology. J Vet Intern Med. 2008;22(4):826-31.

50. Kodre V, Cemazar M, Pecar J, et al., Electrochemotherapy Compared to Surgery for Treatment of Canine Mast Cell Tumours. In Vivo. 2009; 23: 55-62.

51. Skorupski K, Frazier S, et al., Aggressive local therapy combined with systemic chemotherapy provides long-term control in grade II stage 2 canine mast cell tumour: 21 cases (1999-2012). Vet Comp Oncol. 2015; 13(3):267-80.

52. Thompson J, Pearl DL, Yager JA, et al., Canine subcutaneous mast cell tumor: characterization and prognostic indices. Vet Pathol. 2011; 48(1):156-68.

53. Newmana SJ, Mrkonjich L, Walker KK and Rohrbach BW. Canine subcutaneous mast cell tumour: diagnosis and prognosis. Journal of Comparative Pathology 2007; 136: 231-239.

54. Monteiro B, Boston S, Monteith G. Factors influencing complete tumor excision of mast cell tumors and soft tissue sarcomas: A retrospective study in 100 dogs. Can Vet J. 2011; 52(11).

55. Hershey E, Sorenmo K, Hnedrick $M$, et al. Prognosis for presumed feline vaccine-associated sarcoma after excision: 61 cases (1986-1996). JAVMA. 2000; 216(1): 58-61.

56. Selmic $L$ and Ruple A. A systematic review of surgical margins utilized for removal of cutaneous mast cell tumors in dogs. BMC Veterinary Research. 2020; 16:5.

57. Hirsch L, Stafford R, Bankson J, et.al. Nanoshell-mediated near-infrared thermal therapy of tumors under magnetic resonance guidance. Proc Natl Acad Sci U S A 2003 Nov 11;100(23):13549-54

58. Schwartz J, Price R, Gill K. et. al Selective nanoparticle-directed ablation of the canine prostate. Lasers Surg Med, 2011 Mar;43(3):213-20

59. Rastinehad A, Anastos H, Wajswol E et al. Gold nanoshell-localized photothermal ablation of prostate tumors in a clinical pilot device study. Proc Natl Acad Sci U S A. 2019 Sep 10;116(37):18590-18596

60. O'Neal P, Hirsch L, Naomi J Halas et. al Photo-thermal tumor ablation in mice using near infraredabsorbing nanoparticles Cancer Lett. 2004 Jun 25;209(2):171-6 
61. Loo C, Lin A, Hirsch L, et. al. Nanoshell-enabled photonics-based imaging and therapy of cancer. Technol Cancer Res Treat 2004 Feb;3(1):33-40

62. Lal S, Clare S, Halas N. Nanoshell-enabled photothermal cancer therapy: impending clinical impact. Acc Chem Res .2008 Dec;41(12):1842-51

63. Tang Q, Wang Y, Huang R, You Q, et. al. Preparation of anti-tumor nanoparticle and its inhibition to peritoneal dissemination of colon cancer. PLoS One. 2014 Jun 4;9(6)

\section{Tables}

Due to technical limitations, tables are only available as a download in the Supplemental Files section.

\section{Figures}

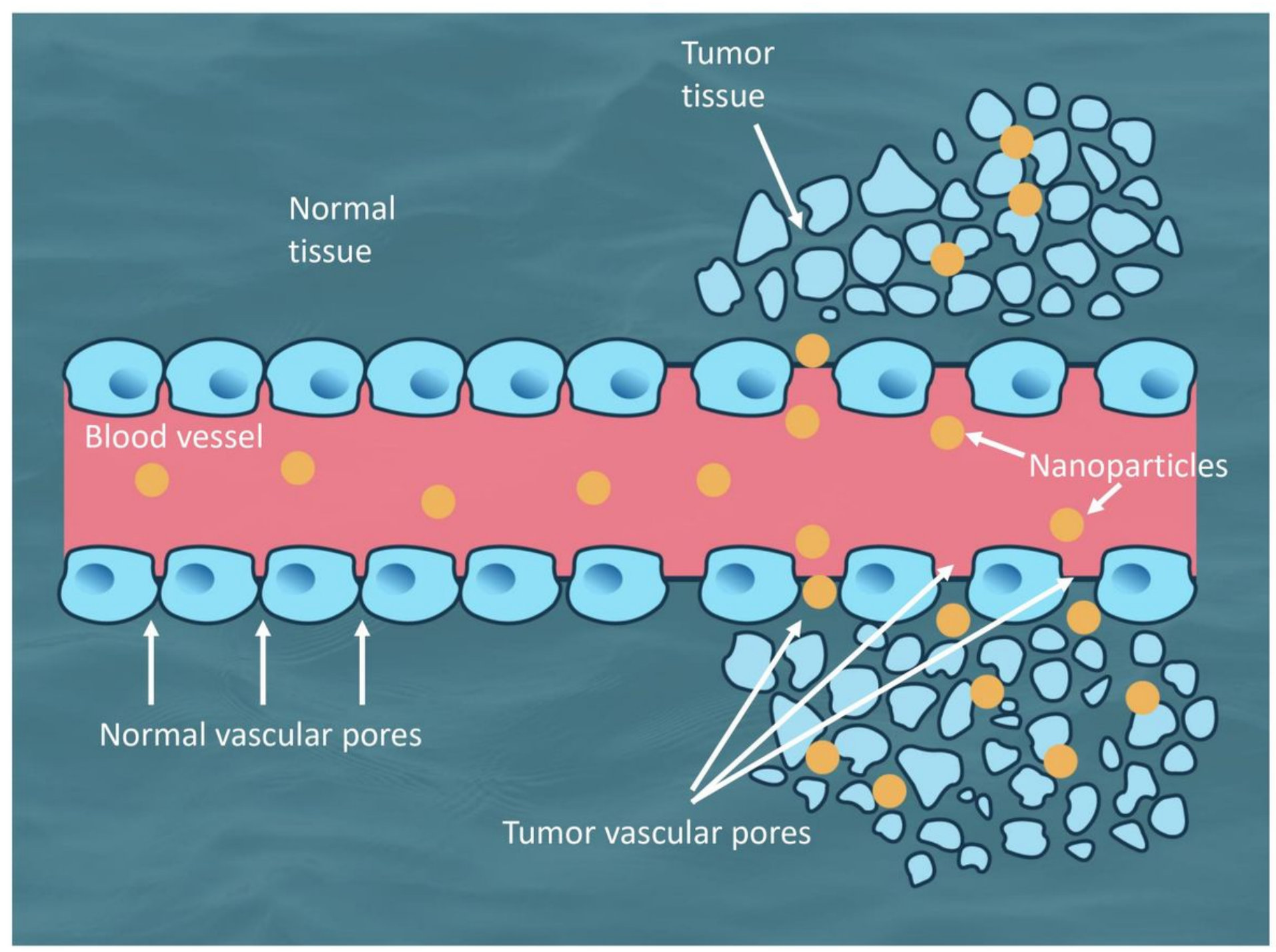

\section{Figure 1}


Graphic demonstration on passive accumulation of nanoparticles through abnormal tumor vasculature and not normal vasculature.

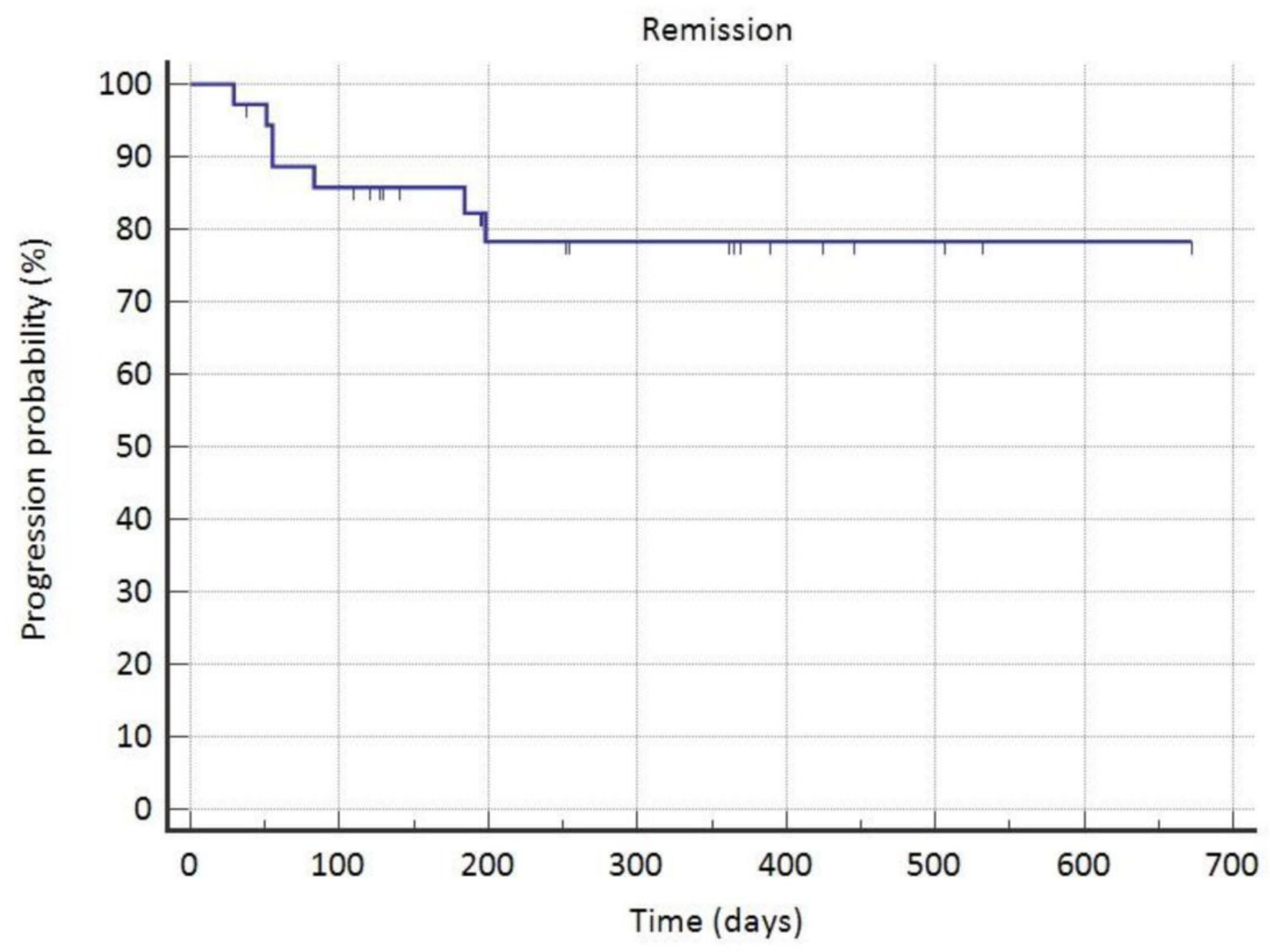

Figure 2

Kaplan Meyer probability curve for the treatment group (blue line). Hash marks indicated date of censoring. 


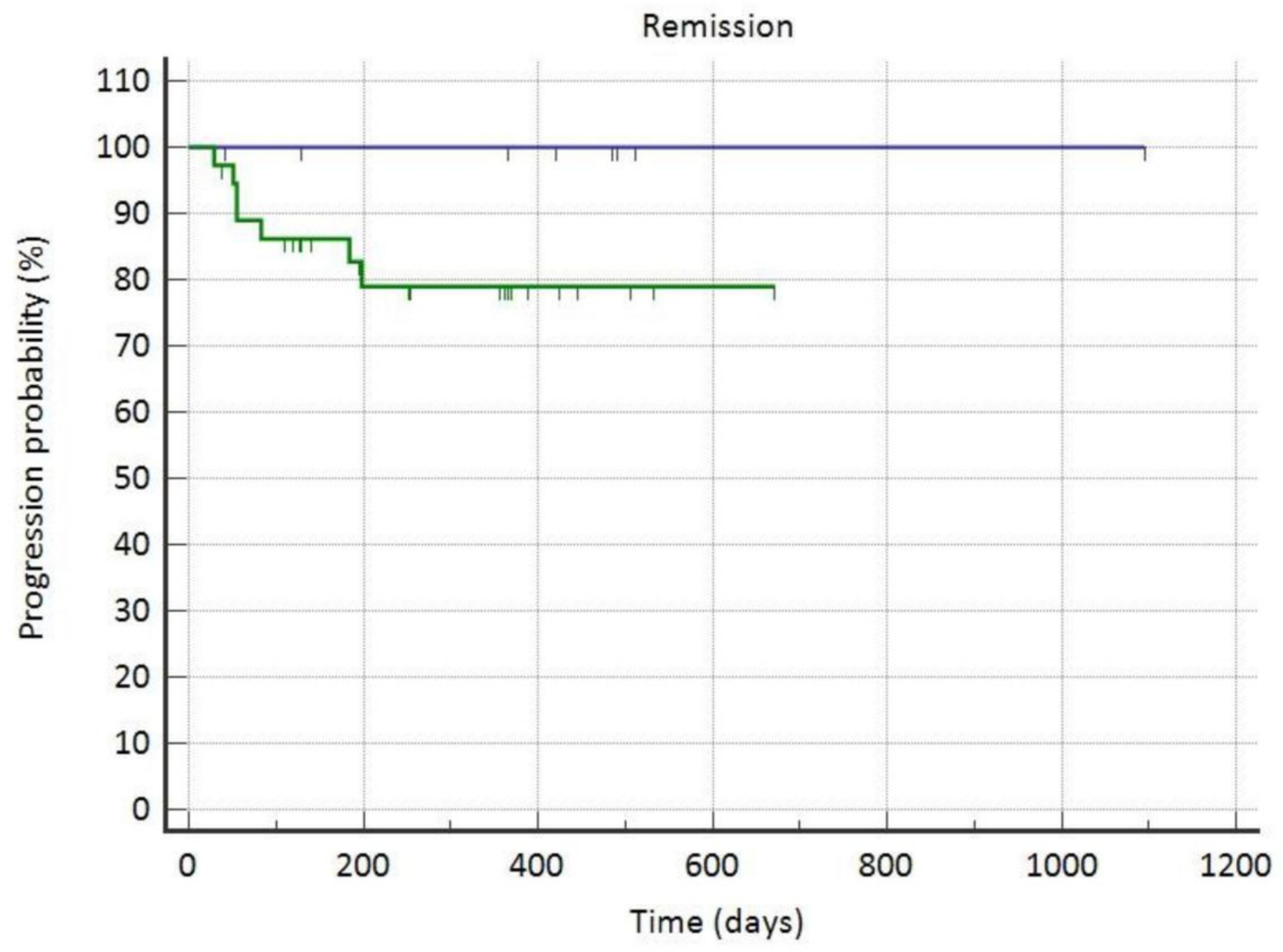

Figure 3

Kaplan Meyer probability curve comparing treatment group (blue line) to control group (green line). Hash marks indicated date of censoring.

\section{Supplementary Files}

This is a list of supplementary files associated with this preprint. Click to download.

- Table1.png

- Table2.png

- Table3.png

- Table4.png 